\title{
Processing Self Corrections in a speech to speech system
}

\author{
Jörg Spilker, Martin Klarner, Günther Görz \\ University of Erlangen-Nuremberg - Computer Science Institute, \\ IMMD 8 - Artificial Intelligence, \\ Am Weichselgarten 9, 91058 Erlangen - Tennenlohe, Germany \\ \{spilker,klarner,goerz\}@immd8. informatik.uni-erlangen.de
}

\begin{abstract}
Speech repairs occur often in spontaneous spoken dialogues. The ability to detect and correct those repairs is necessary for any spoken language system. We present a framework to detect and correct speech repairs where all relevant levels of information, i.c., acoustics, lexis, syntax and semantics can be integrated. The basic idea is to reduce the search space for repairs as soon as possible by cascading filters that involve more and more features. At first an acoustic module generates hypotheses about the existence of a repair. Second a stochastic model suggests a correction for cvery hypothesis. Well scored corrections are inserted as new paths in the word lattice. Finally a lattice parser decides on accepting the repair.
\end{abstract}

\section{Introduction}

Spontaneous spech is disfluent. In contrast to read speech the sentences aren't perfectly planned before they are uttered. Speakers often modify their plans while they speak. This results in pauses, word repetitions or changes, word fragments and restarts. Current automatic speech understanding systems perform very well in small domains with restricted speech but have great difficulties to deal with such disfluencies. A system that copes with these self corrections (=repairs) must recognize the spoken words and identify the repair to get the intended meaning of an utterance. To characterize a repair it is commonly segmented into the following four parts (cf. fig.1):

- reparandum: the "wrong" part of the utterance

- interruption point (IP): marker at the end of the reparandum

- editing term: special phrases, which indicate a repair like "well", "I mean" or filled pauses such as "uhm", "uh"

- reparans: the correction of the reparandum

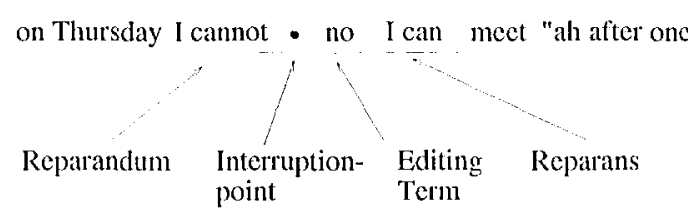

Figure 1: Example of a self repair

Only if reparandum and editing term are known, the utterance can be analyzed in the right way. It remains an open question whether the two terms should be deleted before a semantic analysis as suggested sometimes in the literature ${ }^{1}$. If both terms are marked it is a straightforward preprocessing step to delete reparandum and editing term. In the Verbmobil ${ }^{2}$ corpus, a corpus dealing with appointment scheduling and travel planning, nearly $21 \%$ of all turns contain at least one repair. As a consequence a speech understanding system that cannot handle repairs will lose performance on these turns.

Even if repairs are defined by syntactic and semantic well-formedness (Levelt, 1983) we observe that most of them are local phenomena. At this point we have to differentiate between restarts and other repairs ${ }^{3}$ (modification repairs). Modification repairs have a strong correspondence between reparandum and reparans,

\footnotetext{
${ }^{1}$ In most cases a reparandum could be deleted without any loss of information. But, for example, if it introduces an object which is referred to later, a deletion is not appropriate.

${ }^{2}$ This work is part of the VERBMOBIL project and was funded by the German Federal Ministry for Research and Technology (BMBF) in the framcwork of the Verbmobil Project under Grant BMBF 01 IV 701 V0. The responsibility for the contents of this study lies with the authors.

${ }^{3}$ Often a third kind of repair is defined: "abridged repairs". These repairs consist solely of an editing term and are not repairs in our sensc.
} 
whereas restarts are less structured. In our believe there is no need for a complete syntactic analysis to detect and correct most modification repairs. Thus, in what follows, we will concentrate on this kind of repair.

There are two major arguments to process repairs before parsing. Primarily spontaneous speech is not always syntactically well-formed even in the absence of self corrections. Second (Meta-) rules increase the parsers' search spacc. This is perhaps acceptable for transliterated speech but not for specch recognizers output like lattices because they represent millions of possible spoken utterances. In addition, systems which are not based on a deep syntactic and semantic analysis - e.g. statistical dialog act prediction - require a repair processing step to resolve contradictions like the one in fig. 1.

We propose an algorithm for word lattices that divides repair detection and correction in three steps (cf. fig. 2) First, a trigger indicates potential IPs. Second, a stochastic model tries to find an appropriate repair for each IP by guessing the most probable segmentation. To accomplish this, repair processing is seen as a statistical machine translation problem where the reparandum is a translation of the reparans. For every repair found, a path representing the speaker's intended word sequence is inserted into the laticice. In the last step, a lattice parser selects the best path.

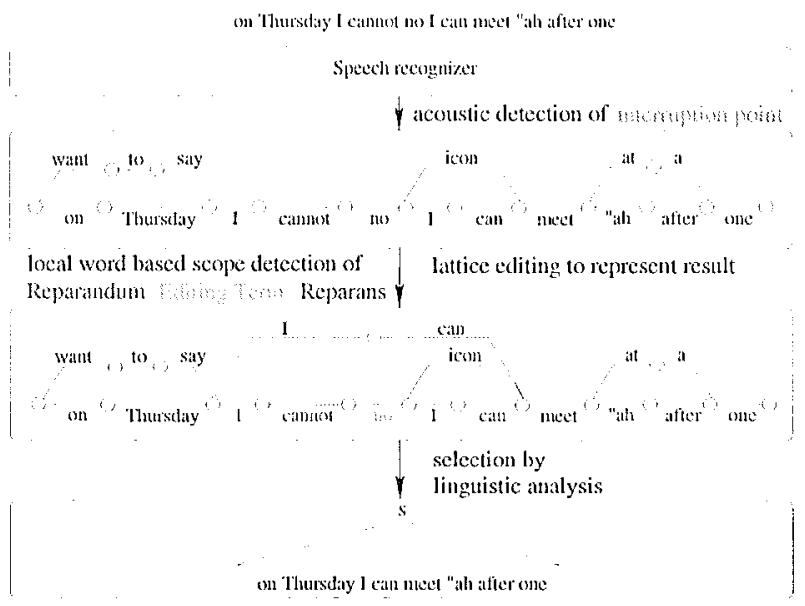

Figure 2: An architecture for repair processing

\section{Repair Triggers}

Because it is impossible for a real time speech system to check for every word whether it can be part of a repair, we use triggers which indicate the potential existence of a repair. These triggers must be immediately detectable for every word in the lattice. Currently we are using two different kind of triggers ${ }^{4}$ :

1. Acoustic/prosodic cues: Speakers mark the IP in many cases by prosodic signals like pauses, hesitations, etc. A prosodic classifier ${ }^{5}$ determines for every word the probability of an IP following. If it is above a certain threshold, the trigger becomes active. For a detailed description of the acoustic aspects see (Batliner et al., 1998).

2. Word fragments are a very strong repair indicator. Unfortunately, no speech recognizer is able to detect word fragments to date. But there are some interesting ap proaches to detect words which are not in the recognizers vocabulary (Klakow et al., 1999). A word fragment is normally an unknown word and we hope that it can be distinguished from unfragmented unknown words by the prosodic classifier. So, currently this is a hypothetical trigger. We will elaborate on it in the evaluation section (cf. sect. 5) to show the impact of this trigger.

If a trigger is active, a search for an acceptable segmentation into reparandum, oditing term and reparans is initiated.

\section{Scope Detection}

As mentioned in the introduction repair scgmentation is based mainly on a stochastic translation model. Before we explain it in detail we give a short introduction to statistical machine translation ${ }^{6}$. The fundamental idea is the assumption that a given sentence $S$ in a source languagc (c.g. English) can be translated in any sentence $\hat{t}$ in a target language (c.g. German). To every pair $(S, \hat{T})$ a probability is assigned which reflects the likelihood that a translator who sees $S$ will produce $\hat{T}$ as the translation. The statistical machine translation problem is

\footnotetext{
${ }^{4}$ Other triggers can be added as well. (Stolcke et al., 1999) for example integrate prosodic cues and an extended language model in a speech recognizer to detect IP's.

${ }^{5}$ The classifier is cleveloped by the speech group of the IMMD 5. Special thanks to Anton Batliner, Richard Iluber and Volker Warnke.

${ }^{6} \Lambda$ more detailed introduction is given by (Brown ct al., 1990)
} 
formulated as:

$$
\hat{T}=\operatorname{argmax}_{T} P(T \mid S)
$$

'This is reformulated by Bayes' law for a better search space reduction, but we are only interested in the conditional probability $P(T \mid S)$. For further processing steps we have to introduce the concept of alignment (Brown et al., 1990). Let $S$ be the word sequence $S_{1}, S_{2} \ldots S_{l} \equiv S_{1}^{l}$ and $T=T_{1}, T_{2} \ldots T_{m} \equiv T_{1}^{m}$. We can link a word in $T$ to a word in $S$. This reflects the assumption that the word in $T$ is translated from the word in $S$. For example, if $S$ is "On Thursday" and $T$ is "Am Donnerstag" "Am" can be linked to "On" but also to "Thursday". If each word in $T$ is linked to exactly one word in $S$ these links can be described by a vector $a_{1}^{m}=a_{1} \ldots a_{m}$ with $a_{i} \in 0 \ldots l$. If the word $T_{j}$ is linked to $S_{i}$ then $a_{j}=i$. If it is not connected to any word in $S$ then $a_{j}=0$. Such a vector is called an alignment $a . P(T \mid S)$ can now be expressed by

$$
P\left(T^{\prime} \mid S\right)=\sum_{\text {a is alignment }} P(T, a \mid S)
$$

Without any further assumptions we can infer the following:

$$
\begin{aligned}
& P(T, a \mid S)= P(m \mid S) * \\
& \prod_{j=1}^{m} P\left(a_{j} \mid a_{1}^{j-1}, T_{1}^{j-1}, m, S\right) * \\
& P\left(T_{j} \mid a_{1}^{j}, T_{1}^{j-1}, m, S\right)
\end{aligned}
$$

Now we return to self corrections. How can this framework help to detect the segments of a repair? Assume we have a lattice path where the reparandum $(R D)$ and the reparans $(R S)$ are given, then $(R S, R D)$ can be seen as a translation pair and $P(R D \mid R S)$ can be expressed cxactly the same way as in equation (2). Hence we have a method to score $(R S, R D)$ pairs. But the triggers only indicate the interruption point, not the complete segmentation. Let us first look at editing terms. We assume them to be a closed list of short phrases. Thus if an entry of the editing term list is found after an IP, the corresponding words are skipped. Any subsequence of words before/after the IP could be the reparandum/reparans. Because turns can have an arbitrary length it is impossible to compute $P(R D \mid R S)$ for every $(R S, R D)$ pair. But this is not necessary at all, if repairs are considered as local phenomena. We restrict our search to a window of four words before and after the IP. A corpus analysis showed that $98 \%$ of all repairs are within this window. Now we only have to compute probabilities for $4^{2}$ different pairs. If the probability of a $(R S, R D)$ pair is above a certain threshold, the scgmentation is accepted as a repair.

\subsection{Parameter Estimation}

The conditional probabilities in equation (3) cannot be estimated reliably from any corpus of realistic size, because there are too many parameters. For example both $P$ in the product; depend on the complete reparans $R S$. Therefore we simplify the probabilities by assuming that $m$ depends only on $l, a_{j}$ only on $j, m$ and $l$ and finally $R D_{j}$ on $R S_{a_{j}}$. So cquation (3) becomes

$$
\begin{aligned}
& P(R D, a \mid R S)=P(m \mid l) * \\
& \quad \prod_{j=1}^{m} P\left(a_{j} \mid j, m, l\right) * P\left(R D_{j} \mid R S_{a_{j}}\right)
\end{aligned}
$$

These probabilities can be directly trained from a manually annotated corpus, where all repairs are labeled with begin, ond, IP and editing term and for each reparandum the words are linked to the corresponding words in the respective reparans. All distributions are smoothed by a simple back-off method (Katz, 1987) to avoid zero probabilities with the exception that the word replacement probability $\left.P(R I)_{j} \mid R S_{a_{j}}\right)$ is smoothed in a more sophisticated way.

\subsection{Smoothing}

Even if we reduce the number of parameters for the word replacement probability by the simplifications mentioned above there are a lot of parameters left. With a vocabulary size of 2500 words, $2500^{2}$ parameters have to be estimated for $P\left(R D_{j} \mid R S_{a_{j}}\right)$. 'The corpus ${ }^{7}$ contains 3200 repairs from which we extract about 5000 word links. So most of the possible word links never occur in the corpus. Somc of them are more likely to occur in a repair than others. For example, the replacement of "Thursday" by "Triday" is supposed to be more likely than by "eating", even if both replacements are not in the training corpus. Of course, this is related to

\footnotetext{
${ }^{7} \sim 11000$ turns with $\sim 240000$ words
} 
the fact that, a repair is a syntactic and/or semantic anomaly. We make use of it by adding two additional knowledge sources to our model. Minimal syntactic information is given by partof-spech (POS) tags and POS sequences, semantic information is given by semantic word classes. Hence the input is not merely a scquence of words but a sequence of triples. Each triple has threc slots (word, POS tag, semantic class). In the next section we will describo how we obtain these two information pieces for every word in the lattice. With this additional information, $P\left(R D_{j} \mid R S_{a_{j}}\right)$ probability could be senoothed by lincar interpolation of word, POS and semantic class replacement probabilities.

$$
\begin{aligned}
& P\left(R D_{j} \mid R S_{a_{j}}\right)= \\
& \quad \alpha * I^{\prime}\left(\operatorname{Word}\left(R D_{j}\right) \mid \operatorname{Word}\left(R S_{u_{j}}\right)\right) \\
& \left.\quad+\beta * I^{\prime}\left(\operatorname{SemClass}(R)_{j}\right) \mid \operatorname{SemClass}\left(R S_{u_{j}}\right)\right) \\
& \quad+\gamma * I^{\prime}\left(\operatorname{POS}\left(R D_{j}\right) \mid P^{\prime} O S\left(R S_{u_{j}}\right)\right)
\end{aligned}
$$

with $\alpha+\beta+\gamma=1$.

Word $\left.(R D)_{j}\right)$ is the notation for the selector of the word slot of the triple at position $j$.

\section{Integration with Lattice Processing}

Wo can now detect, and correct a repair, given a sentence annotated with POS tags and semantic classes. But how can we construct such a sequence from a word latitice? Integrating the model in a lattice algorithm reguires three steps:

- mapping the word lattice to a tag lattice

- triggering IPs and extracting the possible reparandum/reparans pairs

- introducing new paths to represent the plausible reparans

The tag lattice construction is adapted from (Samuelsson, 1997). For every word edge and every denoted POS tag a corresponding tag edge is created and the resulting probability is determined. If a tag edge already exists, the probabilitios of both edges are merged. The original words are stored together with their unique semantic class in a associated list. Paths through the tag graph are scored by a POS-trigram. If a trigger is active, all paths througl the word before the II' need to be tested whether an acceptable repair segmentation exists. Since the scope model takes at most four words for reparandum and reparans in account it is sufficient to expand only partial paths. Wach of these partial paths is then processed by the scope model. 'To reduce the scarch space, paths with a low score can be pruned.

Repair processing is integrated into the Verbmobil system as a filter process between speech recognition and syntactic analysis. This onforces a repair representation that can be integrated into a lattice. It is not possible to mark only the words with some additional information, because a repair is a phenomenon that depends on a path. Imagine that the system has detected a repair on a certain path in the lattice and marked all words by their repair function. 'Then a scarch process (e.g. the parser) selects a different path which shares only the words of the reparandum. But these words are no reparandum for this path. $\Lambda$ solution is to introduce a new path in the lattice where reparandum and editing terms are deleted. As we said before, we do not want to delete these segments, so they are stored in a special slot of the first word of the reparans. The original path can now be reconstruct if necessary.

To ensure that these new paths are comparable to other paths we score the reparandum the same way the parser does, and add the resulting value to the first word of the reparans. $\Lambda$ s a result, both the original path and the one with the repair get the same score except one word transition. The (probably bad) transition in the original path from the last word of the reparandum to the first word of the reparans is replaced by a (probably good) transition from the reparandum's onset to the reparans. We take the lattice in fig. 2 to give an example. The scope model has marked "I cannot" as the reparandum, "no" as an editing term, and "T can" as the reparans. We sum up the acoustic scores of "l", "can" and "no". Then we add the maximum language model scores for the transition to "l", to "can" given "l", and to "no" given "l" and "can". This score is added as an offset to the acoustic score of the second "I".

\section{$5 \quad$ Results and Further Work}

Due to the different trigger situations we performed two tests: One where wo use only acoustic triggers and another where the existence of a perfect, word fragment detector is assumed. The input were unsegmented transliterated utterance to exclude influences a word 
recognizer. We restrict the processing time on a SUN/ULTRA 300MHZ to 10 seconds. The parser was simulated by a word trigram. Training and testing were done on two separated parts of the German part of the Verbmobil corpus (12558 turns training / 1737 turns test).

\begin{tabular}{|r|r|r|r|r|}
\hline & \multicolumn{2}{|c|}{ Detction } & \multicolumn{2}{c|}{ Correct scope } \\
\hline & Recall & Precision & Recall & Precision \\
\hline Test 1 & $49 \%$ & $70 \%$ & $47 \%$ & $70 \%$ \\
\hline Test 2 & $71 \%$ & $85 \%$ & $62 \%$ & $83 \%$ \\
\hline
\end{tabular}

A direct comparison to other groups is rather difficult due to very different corpora, evaluation conditions and goals. (Nakatani and Hirschberg, 1993) suggest a acoustic/prosodic detector to identify IPs but don't discuss the problem of finding the correct segmentation in depth. Also their results are obtained on a corpus where every utterance contains at least one repair. (Shriberg, 1994) also addresses the acoustic aspects of repairs. Parsing approaches like in (Bear et al., 1992; Hindle, 1983; Core and Schubert, 1999) must be proved to work with lattices rather than transliterated text. An algorithm which is inherently capable of lattice processing is proposed by Hecman (Hecman, 1997). He redefines the word recognition problem to identify the best sequence of words, corresponding POS tags and special repair tags. He reports a recall rate of $81 \%$ and a precision of $83 \%$ for detection and $78 \% / 80 \%$ for correction. The test settings are nearly the same as test 2. Unfortunatcly, nothing is said about the processing time of his module.

Wo have presented an approach to score potential reparandum/reparans pairs with a relative simple scope model. Our results show that repair processing with statistical methods and without deep syntactic knowledge is a promising approach at least for modification repairs. Within this framework more sophisticated scope models can be evaluated. A system integration as a filter process is described. Mapping the word lattice to a POS tag lattice is not optimal, because word information is lost in the search for partial paths. We plan to implement a combined combined POS/word tagger.

\section{References}

A. Batliner, R. Kompe, A. Kießling, M. Mast, H. Niemann, and E. Nöth. 1998. $\mathrm{M}=$ syntax + prosody: A syntactic-prosodic la- belling schema for large spontaneous speech databases. Speech Communication, 25:193222.

J. Bear, J. Dowding, and E. Shriberg. 1992. Integrating multiple knowledge sources for detection and correction of repairs in human computer dialogs. In Proc. $A C L$, pages 56-63, University of Delaware, Newark, Delaware.

P. F. Brown, J. Cocke, S. A. Della Pietra, V. J. Della Pietra, F. Jelinek, J. D. Lafferty, R. L. Mercer, and P. S. Roossin. 1990. A statistical approach to machine translation. Computational Linguistics, 16(2):79-85, June.

M. G. Core and K. Schubert. 1999. Speech repairs: A parsing perspective. Satellite mecting ICPIIS 99.

P. A. Heeman. 1997. Speech Repairs, Intonation Boundaries and Discourse Markers: Modeling Speakers' Utterances in Spoken Dialog. Ph.D. thesis, University of Rochester.

D. Hindle. 1983. Deterministic parsing of syntactic nonfluencies. In Proc. $A C L$, MIT, Cambridge, Massachusetts.

S. M. Kat\% 1987. Estimation of probabilities from sparse data for the language model component of a specch recognizer. Transaction on Acoustics, Speech and Signal Processing, ASSP-35, March.

D. Klakow, G Rose, and X. Aubert. 1999. OOV-Detection in Large Vocabulary System Using Automatically Defined WordFragments as Fillers. In EUROSPELCH' 99 , volume 1, pages 49-52, Budapest.

W. Levelt. 1983. Monitoring and self-repair in speech. Cognition, 14:41-104.

C. Nakatani and J. Hirschberg. 1993. A speechfirst model for repair detection and correction. In Proc. ACL, Ohio State University, Columbus, Ohio.

C. Samuelsson. 1997. A left-to-right tagger for word graphs. In Proc. of the 5th International workshop on Parsing technologies, pages 171178, Bosten, Massachusetts.

E. E. Shriberg. 1994. Preliminaries to a Theory of Speech Disflucncies. Ph.D. thesis, University of California.

A. Stolcke, E. Shriberg, D. Hakkani-Tur, and G. Tur. 1999. Modeling the prosody of hidden events for improved word recognition. In EUROSPEECH '99, volume 1, pages 307310, Budapest. 